\title{
In-Cell Protease Assay Systems Based on Trans-Localizing Molecular Beacon Proteins Using HCV Protease as a Model System
}

\author{
Jeong Hee Kim ${ }^{1 *}$, Min Jun Lee ${ }^{1,3}$, Inhwan Hwang ${ }^{2}$, Hyun Jin Hwang ${ }^{3 *}$ \\ 1 Department of Biochemistry and Molecular Biology, School of Dentistry, Kyung Hee University, Seoul, Korea, 2 Department of Life Science, POSTECH, Pohang, Korea, \\ 3 R\&D Center, Ahram Biosystems Inc., Seoul, Korea
}

\begin{abstract}
This study describes a sensitive in-cell protease detection system that enables direct fluorescence detection of a target protease and its inhibition inside living cells. This live-cell imaging system provides a fluorescent molecular beacon protein comprised of an intracellular translocation signal sequence, a protease-specific cleavage sequence, and a fluorescent tag sequence(s). The molecular beacon protein is designed to change its intracellular localization upon cleavage by a target protease, i.e., from the cytosol to a subcellular organelle or from a subcellular organelle to the cytosol. Protease activity can be monitored at the single cell level, and accordingly the entire cell population expressing the protease can be accurately enumerated. The clear cellular change in fluorescence pattern makes this system an ideal tool for various life science and drug discovery research, including high throughput and high content screening applications.
\end{abstract}

Citation: Kim JH, Lee MJ, Hwang I, Hwang HJ (2013) In-Cell Protease Assay Systems Based on Trans-Localizing Molecular Beacon Proteins Using HCV Protease as a Model System. PLoS ONE 8(3): e59710. doi:10.1371/journal.pone.0059710

Editor: Francesco Pappalardo, University of Catania, Italy

Received December 10, 2012; Accepted February 17, 2013; Published March 21, 2013

Copyright: $\odot 2013 \mathrm{Kim}$ et al. This is an open-access article distributed under the terms of the Creative Commons Attribution License, which permits unrestricted use, distribution, and reproduction in any medium, provided the original author and source are credited.

Funding: This study was supported by a grant from the Ministry of Health and Welfare (03-PJ1-PG11-VN01-SV02-0019). The funders had no role in study design, data collection and analysis, decision to publish, or preparation of the manuscript.

Competing Interests: Ahram Biosystems, Inc. has no competing interest. All authors confirm that this manuscript does not alter the authors' adherence to all the PLOS ONE policies on sharing data and materials.

* E-mail: jhkimh@khu.ac.kr (JHK); ahrambio@gmail.com (HJH)

\section{Introduction}

Proteases are involved in many critical biological pathways and have been the focus of a broad range of biological and diseaserelated process studies, including apoptosis, Alzheimer's disease and viral infections [1-4]. Inhibition or inactivation of a specific protease can inhibit or block cellular processes that are induced by that protease, and these proteases became attractive targets for drug development. Accordingly, there has been significant interest in developing new technologies for monitoring the activity of a target protease and its inhibition inside a living cell [5-6]. Compared to biochemical methods, cell-based assays have become an important part of the pre-clinical drug discovery process because cellular integrity and toxicity can be monitored at the time of performing the target assay. These advantages encourage development of cell-based assay for various targets in the cell [69]. The primary cell-based assays involved cell surface cutting or cell fixation [9-10] because developing technology that enables direct observation of live cells, and the targets within them, has been challenging. In this study, we developed in-cell protease assay systems based on molecular beacon reporter (MBR) proteins with intracellular trans-localizing properties that are modulated depending on the action or inaction of a target protease inside a living cell. The MBR proteins were designed to exhibit different trans-localization before and after a protease-induced cleavage. We demonstrate the effectiveness of these novel in-cell protease assay systems using the hepatitis $\mathrm{G}$ virus (HCV) NS3 protease and its cleavage sequence (CS) as a model system.
The MBR proteins used in this study were constructed to contain an NS3 serine protease-specific CS, an intracellular translocation signal sequence $(\mathrm{s})$, and a fluorescent protein $(\mathrm{s})$ to detect translocation of the fluorescent protein following NS3 protease-specific cleavage inside a living cell. Two types of MBR proteins were formulated: type I which shows translocation of a fluorescent protein from a subcellular organelle to the cytosol upon cleavage, and type II, which exhibits translocation of a fluorescent protein from the cytosol to a subcellular organelle upon cleavage. Using these MBR proteins, we have demonstrated that the mechanism and level of protease activity can be monitored at the single-cell level. Therefore, the level of the protease activity can be accurately enumerated in an entire cell population. Because the fluorescent image change of the cells can be clearly and easily monitored, this novel method is an ideal tool for biological and drug discovery researchers.

\section{Materials and Methods}

Cell Culture, Drug Treatment and Cytotoxicity Analysis

Chinese hamster ovary (CHO-K1, ATCG CCL-17) cells were cultured in appropriate media as recommended by the supplier. Exponentially growing cells were seeded at $5 \times 10^{5}$ cells/well in a six-well plate and treated to test the in-cell protease assay systems as described in the Results section. 


\begin{tabular}{l|c|c|c|c|c|}
\cline { 2 - 5 } Type I & TS & CS & \multicolumn{2}{|c|}{ GFP } \\
\hline $\begin{array}{l}\text { Type II } \\
\text { Mono-color }\end{array}$ & GAPDH & CS & TS & GFP \\
\cline { 2 - 5 } $\begin{array}{l}\text { Type II } \\
\text { Dual-color }\end{array}$ & GFP & CS & TS & RFP \\
\cline { 2 - 5 } & \multicolumn{5}{|c}{}
\end{tabular}

Figure 1. Schematic illustration of MBR proteins used in this study. One type I and two different type II MBRs were constructed. Either GAPDH or GFP was used as a masking protein for mono-color or dual-color type II MBR, respectively. TS; translocation signal, CS; cleavage sequence, GFP; green fluorescent protein, GAPDH; glyceraldehydes 3-phosphate dehydrogenase. RFP; red fluorescent protein. doi:10.1371/journal.pone.0059710.g001

\section{Construction and Preparation of Plasmids}

Plasmids encoding several substrate chimeric MBR proteins were constructed. For the construction of the type I substrate, green fluorescent protein (GFP) was amplified with primers $5^{\prime}$ GCG GGT ACG ATG GTG AGC AAG GGC GAG-3' and 5'GCG GAA TTG CTT GTA CAG CTG GTG CAT-3' and cloned into the pcDNA3.1 vector (Invitrogen, USA). Subsequently, the proteolytic CS of the NS3 HCV protease was inserted between GFP and the Pleckstrin homology (PH) domain by polymerase chain reaction (PCR) amplification using primers $5^{\prime}$ GCG GAA TTC GAG GCC AAC GCG GAG GAT GTC GTG TGG TGG TGA ATG TCT TAC TCT TGG ACA GGC GCA CTC ATC GAT GAC TCG GGT AGG GAC TTC-3' and 5'GCG TCT AGA TCA CTG GAT GTT GAG GTC-3'. The resulting plasmid was named $\mathrm{pHCV}-\mathrm{CS} \mathrm{a} / \mathrm{Ib}-\mathrm{GFP}$.

For the type II substrate, two constructs, mono-color type II and dual-color type II were prepared. Glyceraldehydes-3-phosphate dehydrogenase (GAPDH) or GFP was used as a masking protein for mono-color or dual-color type II MBR proteins, respectively. To clone GAPDH, total RNA was prepared from CHO-K1 cells by using Trizol (MRC, USA) and cDNA was produced with reverse transcriptase (Invitrogen, USA). Complementary DNA for GAPDH was amplified with primers 5'-GCG GGT ACG ATG GGG AAG GTG AAG GTC GGA GTC-3' and 5'-GCG GAA TTG CTC CTT GGA GGG CAT GTG GGG CAT-3' and cloned into pcDNA3.1. Mitochondrial targeting sequence of methionine sulfoxide reductase (MSRA) was placed at the Nterminus of GFP by amplification using the primers 5'-GCG GAA TTC ATG CTC TCG GCC ACG CGG AGG GCT TGC CAG

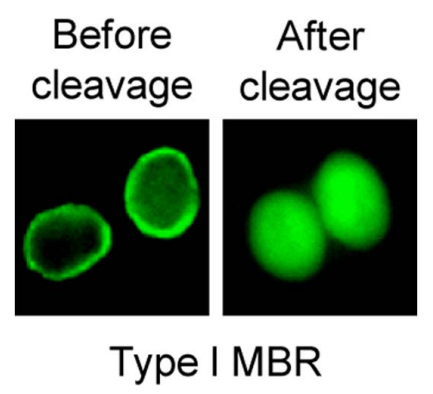

Figure 2. Detection of HCV NS3 protease using type I MBR. Before cleavage, GFP was found in the plasma membrane (ring shape, before cleavage). Cleavage of the type I MBR by HCV NS3 protease resulted in the translocation of GFP to the cytosol (dispersed image, after cleavage).

doi:10.1371/journal.pone.0059710.g002
GTC CTG CTG GTG GAC AGG CTG TTT CGC GTG CGG AGG ATG GAC TCG GGT AGG GAC TTC-3' and 5'-GGG TGT AGA TTA GTT GTA GAG CTC GTC CAT GGG-3'. The proteolytic CS of NS3 HCV protease was placed at the Nterminus of the mitochondrial targeting sequence by PCRamplification using the primers 5'-GCG GAA TTC GAG GCG AAG GGG GAG GAT GTC GTG TGG TGG TCA ATG TCT TAC TCT TGG ACA GGC GCA GTC ATG CTC TGG GCG ACG CGG AGG-3' and 5'-GGG GAA TTG GAG GAT GTC GTG TGG TGG TGA ATG TCT TAC ATG CTG TGG GGG ACG CGG AGG-3' (CS:MSRA:GFP). Finally, the GAPDH sequence was placed at the N-terminus of the CS:MSRA:GFP clone without a stop codon to generate the plasmid, pHCV-CSla/ IIa-GFP.

To clone dual-color type II MBR (3.1-GFP:CS1a(10:10):MSRA:RFP), the mitochondrial targeting sequence of MSRA was fused to RFP (MSRA:RFP) by PGR amplification with primers 5'GCG GAA TTC ATG CTC TCG GCG ACG CGG AGG GCT TGG CAG CTC CTG CTC GTC CAC AGC GTG TTT CGG GTC CGG AGG ATG GTG CGG TCG TCG AAG AAC-3' and 5'-GCG TCT AGA TTA CAG GAA CAG GTG GTG-3'. Next, the CS for HCV NS3 was placed in frame to generate CS:MSRA:RFP. For this purpose, primers 5'-GCG GAA TTC GAG GCG AAC GCG GAG GAT GTC GTG TGC TGC TCA ATG TGT TAG TCT TGG ACA GGG GGA GTC ATG GTC TCG GCG ACG CGG AGG-3' And 5'-GGG TCT AGA TTA CAG GAA CAG GTG GTG-3' were used. Finally, this construct was subcloned into a GFP containing vector at the Eco RI and $X b a$ I sites. To generate plasmids expressing different sizes of CS, appropriate primers were prepared by the shortening above described primers to generate specified sequences, as described in the text. The HCV protease clone used in this study, pHCV-NS3/ NS4A contained NS3 and NS4a domains for its enzymatic activity [11-13].

\section{Transfection and Observation of Intracellular the Protease Activity}

Plasmid DNAs coding for the HCV NS3 protease and its substrate protein were prepared using the RPM turbo and/or maxiprep kits (Q-Biogene, USA) and transfected into 50\% confluent CHO-K1 cells using the Gene Shuttle 40 kit (QBiogene, USA). This kit was also used to deliver the RNA aptamer into the cells for the inhibition tests. Cells were seeded into 12 well plates and transfected with plasmids coding for NS3 protease and its substrate MBR protein. The cells were observed under a fluorescent microscope (Nikon E800, Japan) at the appropriate time points after transfection. Cells exhibiting (i.e., ring-like shape for type I MBR or a dispersed pattern for type II MBR) or not exhibiting protease activity (i.e., dispersed pattern for type I MBR or speckles for type II MBR) were counted. At least three different experiments were performed for each assay and at least 500 cells were counted for each experiment. The relative percent cleavage was calculated as the number of cells exhibiting HCV protease inhibition divided by the total number of cells counted.

\section{Western Blot Analysis}

After transfection, cell lysates were prepared as described previously [14] and the protein concentration was determined. After sodium dodecyl sulfate-polyacrylamide gel electrophoresis (SDS_PAGE), proteins were transferred to polyvinylidene difluoride (PVDF) membranes (Millipore, USA) for $2 \mathrm{hr}$ at $80 \mathrm{~mA}$. Blots were probed with a mouse monoclonal anti-GFP (BD Clontech, USA), Immunoreactivity was detected using either a peroxidaseconjugated anti-mouse igG (Santa Cruz Biotechnology, USA) or 
A
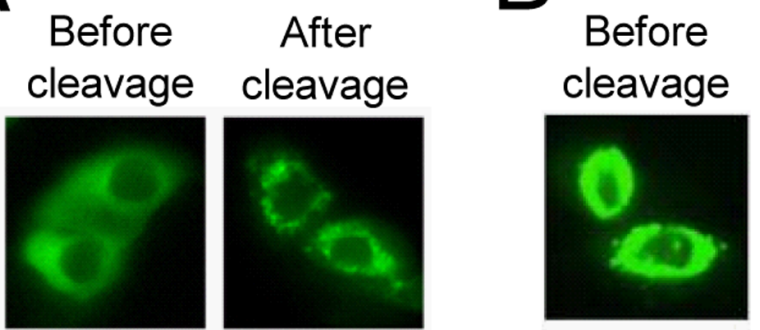

After
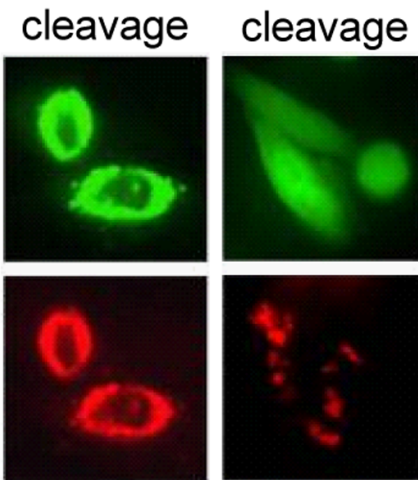

GFP
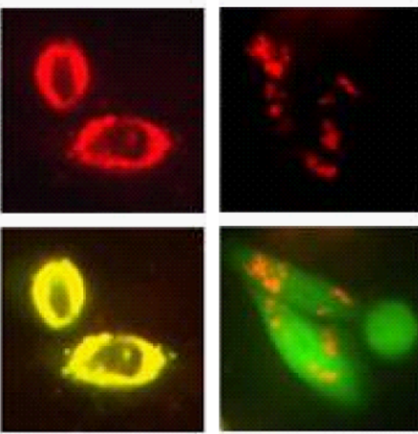

RFP

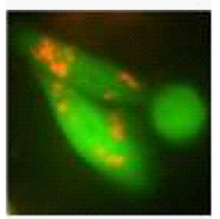

Merged

\section{Mono-color Type II MBR}

\section{Dual-color \\ Type II MBR}

Figure 3. Detection of HCV NS3 protease using type II MBRs. Type II MBRs were constructed to contain a mitochondrial targeting sequence, MSRA as a masked translocation signal. The masking protein used was GAPDH or GFP for mono- or dual-color type II MBRs, respectively. A. Cleavage of mono-color type II MBR by HCV NS3 protease activates the mitochondrial translocation signal of MSRA, resulting in translocation of GFP from the cytosol (before cleavage) to the mitochondria (after cleavage). B. Cleavage of dual-color type II MBR which contains both GFP and RFP, results in translocation of the RFP from the cytosol (before cleavage) to mitochondria while GFP remains in the cytosol (after cleavage) following cleavage. GFP; green fluorescent protein, RFP; red fluorescent protein.

doi:10.1371/journal.pone.0059710.g003

anti-rabbit IgG (Amersham, UK) peroxidase-conjugated secondary immunoglobulin $\mathrm{G}$ antibody followed by enhanced chemiluminescence kit (ECL, Amersham, UK). Experiments were repeated at least three times.

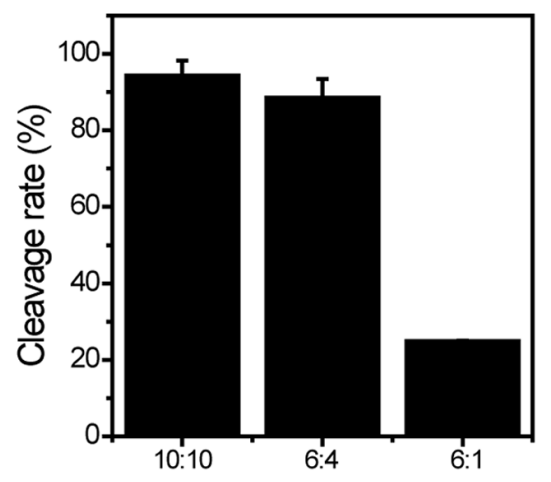

Cleavage sequence size (amino acids)

Figure 4. Cleavage sequence length-dependent cleavage efficiency of MBR protein. The cleavage sequence length examined were 20 (10 amino acids-cleavage site-10 amino acids), 10 (6 amino acids-cleavage site- 4 amino acids), and 7 (6 amino acid-cleavage site- 1 amino acid). Cleavage efficiency was measured using type I MBR and the data was plotted. Experiments were performed at least three times and the data is presented as mean \pm standard deviation (SD). doi:10.1371/journal.pone.0059710.g004

\section{Inhibition of HCV Protease}

A known inhibitor of the HCV NS3 protease, Ac-Asp-D-GlaLeu-Ile-b-cyclohexyl-Ala-Cyc-OH (N-1725.0001, Bachem, Germany) was purchased and dissolved in dimethyl sulfoxide. The stock solution was stored at $-20^{\circ} \mathrm{C}$ and diluted in phosphatebuffered saline to the appropriate working concentration before addition to the cell culture medium during transfection process.

The HCV NS3 protease inhibitory RNA aptamer (G9-I) was prepared as described previously [15]. Briefly, we synthesized a single-stranded DNA template for the HCV NS 3 protease inhibitor template (NS3 I-temp), 5'-GGG AGA ATT CGG ACG AGA AGG TTC GGG ATT TGA GGG TAG AAT GGG AGT ACG TTT GCT GTG TCG TTG CTG TTG T-3', using a DNA synthesizer (Expedite 8900 Nucleic Acid Synthesis System, Applied Biosystems, USA). This template was converted to a double stranded DNA molecule by DNA polymerization with the NS3 I-3' primer 5'-AGA AGA GGA AGG AGA GAG GAA AGG-3'. The double stranded DNA was amplified by PCR using NS3 I-3' and NS3 I-5'T7 (5'-AGT AAT AGG ACT CAC TAT AGG GAG AAT TCG GAC CAG AAG-3') primers, purified with phenol, precipitated with ethanol and recovered by centrifugation. The RNA aptamer was produced from the PCR product by in vitro transcription using the RiboMAX kit (Promega, USA)., and added to cells at a concentration of approximately $7.5 \mu \mathrm{g} / \mathrm{mL}$ during transfection.and the cells were incubated until fluorescence image analysis. 


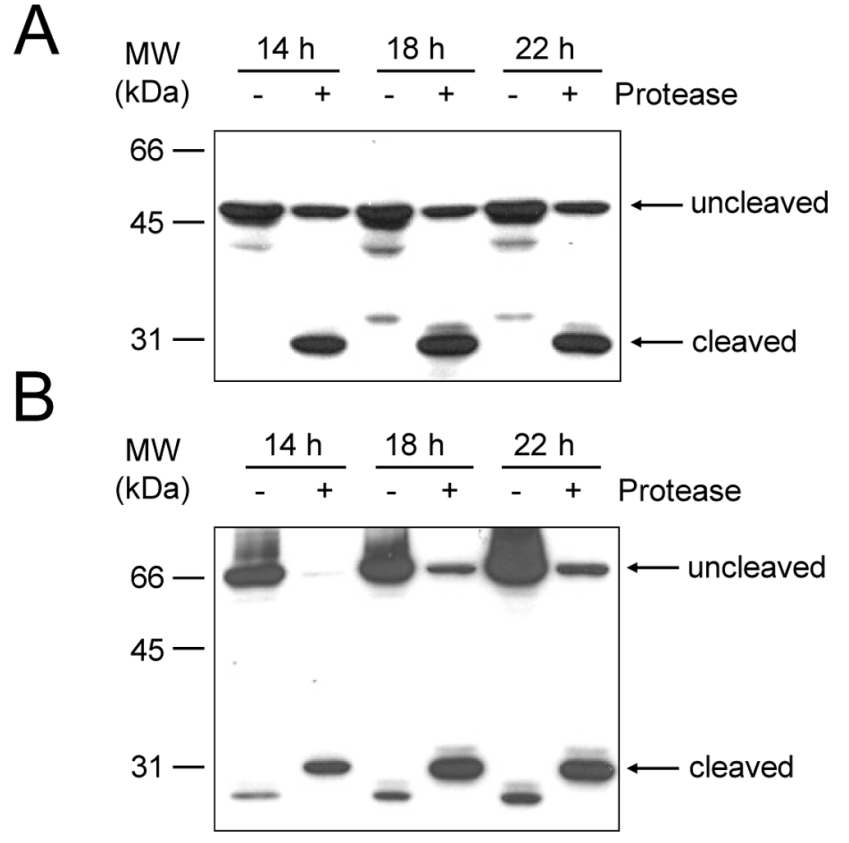

Figure 5. Western blot analysis of MBR protein cleavage. Type I (A) and type II (B) MBR proteins were expressed alone (-; lanes 1, 3, and 5 for $A$ and $B$ ) or with protease (+; lanes 2,4 , and 6 for $A$ and B). Cell lysates prepared after the indicated incubation times were subjected to immunoblotting with an anti-GFP antibody, as described in the Materials and Methods. A. The expected size of $50.2 \mathrm{kDa}$ (uncleaved) and $27.4 \mathrm{kDa}$ (cleaved) were observed with type I MBR protein. B. The expected size of $60.7 \mathrm{kDa}$ (uncleaved) and $27.4 \mathrm{kDa}$ (cleaved) were observed with mono-color type II MBR protein. doi:10.1371/journal.pone.0059710.g005

\section{Results}

Construction of Plasmids Expressing MBR Proteins and their Subcellular Translocation Upon Cleavage by HCV Protease

MBR proteins used in this study are shown in Fig. 1. Two types of MBRs, type I and type II were constructed. Type I MBR contains an $\mathrm{N}$-terminal $\mathrm{PH}$ domain as a translocation signal to the plasma membrane [16-17], a CS recognized by the HCV NS3 protease and GFP at the C-terminal. Thus the tagging fluorescent protein of type I MBR is designed to change its subcellular localization from a subcellular organelle, the plasma membrane, to the cytosol upon cleavage by HCV protease.

Type II MBRs contain a masking protein, CS, translocation signal and a fluorescent protein. Two different subtypes of type II MBR were generated; mono-color and dual-color. For the monocolor MBR, GAPDH was used as a masking protein and GFP was used as a fluorescent tag that changes its subcellular location upon cleavage by the protease. The mitochondrial targeting sequence of MSRA was used as a translocation signal [18-19] and was placed in between the masking protein and the tagging fluorescent protein. For the dual-color MBR, GFP was used as a masking protein and RFP was used as the tagging fluorescent protein. In this case, the tagging fluorescent protein was designed to redistribute the type II MBR from the cytosol to another subcellular organelle, in this case, the mitochondria after cleavage by $\mathrm{HCV}$ protease.

First, the expression and subcellular translocation of the MBRs created were observed. Since they contain a plasma membranetargeting domain, type I MBR proteins and thus GFP, should be directed to the plasma membrane following transfection. As expected, GFP was observed in the plasma membrane as a ringshape in living cells (Fig. 2, left, before cleavage). When type I MBR was co-expressed with $\mathrm{HCV}$ NS3 protease, GFP was observed in the cytosol in a dispersed pattern (Fig. 2, right, after cleavage). This result indicated that HCV NS3 protease cleaved the type I MBR, thereby changing the subcellular location of GFP from the plasma membrane to the cytosol. These changes in fluorescence localization were clearly observed without significant alterations to cell integrity.

Next, we evaluated the fluorescence re-distribution of type II MBRs. The translocation signal of these proteins was blocked by a masking protein at the N-terminus. Before cleavage, mono-color type II MBR resides in the cytosol because the masking protein, GAPDH directs the localization of the MBR protein. Thus, GFP was dispersed throughout the cytosol (Fig. 3A, left, before cleavage). When it was cleaved by the co-expressed HCV NS3 protease, the mitochondrial targeting sequence was revealed at the
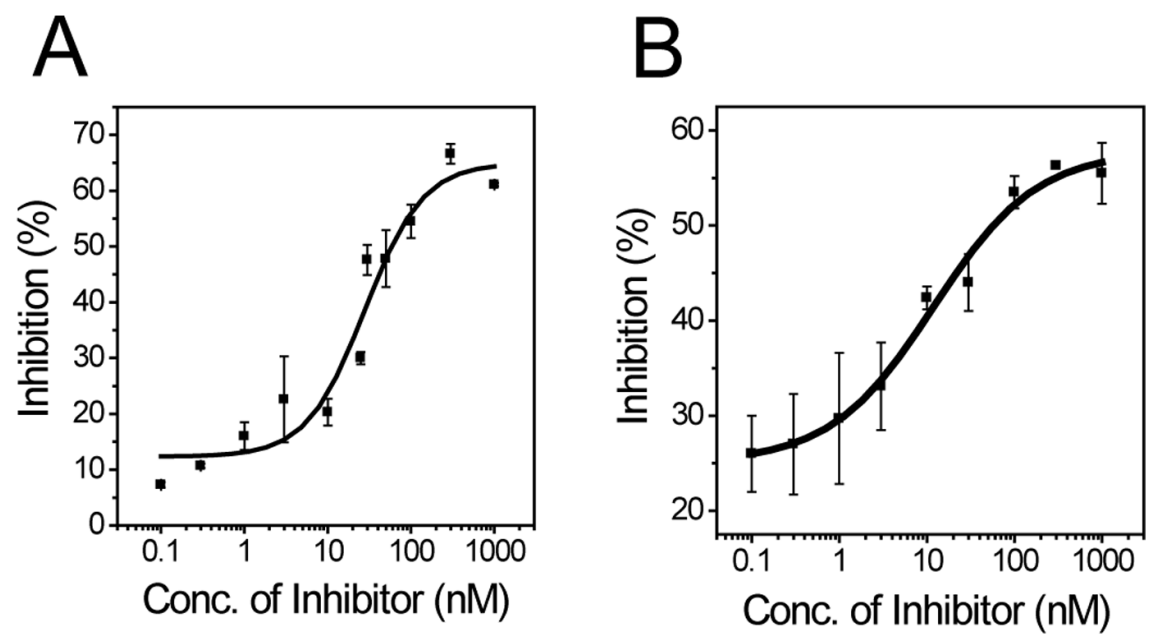

Figure 6. Inhibition of HCV NS3 protease by a known inhibitor. The cleavage of type I MBR (A) and type II MBR (B) by HCV NS3 protease was monitored in the presence of a known peptide derivative inhibitor, Ac-Asp-D-Gla-Leu-lle- $\beta$-cyclohexyl-Ala-Cyc-OH. Both type I and type II MBRs exhibited significant dose-response at nanomolar concentrations. doi:10.1371/journal.pone.0059710.g006 
N-terminus, thereby directing the MBR protein to the mitochondria. Thus, GFP localization changed to the mitochondria and it was observed as small speckles as expected (Fig. 3A, right, after cleavage). When dual-color type II MBR protein was expressed, MBR protein was expressed in the cytosol. Therefore, both GFP and RFP were observed in the cytosol as overlapping images. (Fig. 3B, left, before cleavage). Upon cleavage of dual-color type II MBR, the mitochondrial targeting sequence was revealed at the $\mathrm{N}$-terminus, thereby targeting RFP to the mitochondria while maintaining GFP in the cytosol. As expected, RFP was observed in the mitochondria as small speckles and the green fluorescence was observed as a dispersed image in the cytosol (Fig. 3B, right, after cleavage). The fluorescence re-distribution of both mono- and dual-color type II MBRs was clearly observed in living cells with no significant effect on cell integrity. Similar results were obtained with different cell lines such as human embryonic kidney 293 cells (data not shown). These results demonstrate that the MBR proteins constructed in this study function exactly as designed and therefore can be used as a model assay system for HCV inhibitor screening.

\section{Comparison of Cleavage Sequences in MBR Proteins}

The consensus sequence of the trans-cleavage sites recognized by HCV NS3 protease is D/EXXXXC-A/S, where $\mathrm{X}$ represents any amino acid and the scissile bond is located between $\mathrm{C}$ and $\mathrm{A} /$ $\mathrm{S}$ [9]. In order to optimize cleavage efficiency we compared the cleavage efficiency of the MBR constructs with various CS length, including EANAEDVVGC-SMSYSWTGAL (10:10), EDVVGCSMSY (6:4), and. EDVVCG-S (6:1). These three CS's were designed to contain conserved amino acid sequences. Type I MBRs containing these three different CS's were constructed and tested for cleavage efficiency. The number of cells with ring-shape fluorescence (uncleaved MBR) in the plasma membrane and cells with dispersed fluorescence in the cytosol (cleaved MBR) were counted to determine the MBR cleavage efficiency. As shown in Fig. 4, MBRs that contained 10 amino acids each before and after the CS (10:10) as well as those that contained 6 amino acids before and 4 amino acids after the C.S (6:4) displayed higher cleavage efficiency at $94 \%$ and $89 \%$, respectively. MBRs that contained 6 amino acids before and 1 amino acid after the CS (6:1) showed approximately $25 \%$ cleavage. The same experiment was performed with type II MBRs and yielded similar results (data not shown). These results suggest that the optimum CS size with sufficient cleavage efficiency needs to be approximately equal to or greater than 10 amino acids in length with conserved amino acid sequence. We used C.S (6:4) in MBR proteins from this point forward.

\section{Confirmation of MBR Protein Cleavage by Western Blot Analysis}

The cleavage of MBR proteins was confirmed by Western blot analysis. Cells were transfected with vectors expressing either type I or type II MBRs. Another group of cells were co-transfected with vectors containing MBR proteins and HCV NS3 protease. Cells from both groups were harvested after the indicated period of incubation, and lysates were separated on SDS-PAGE, blotted onto PVDF membrane, and probed with anti-GFP antibody.

As shown in Fig. 5, the expressed MBR proteins were identified with the expected molecular weights. When MBR proteins were co-expressed with the HCV protease, the detectable MBR fragments were smaller in size as expected. Type I MBR was a $50.2 \mathrm{kDa}$ protein (Fig. 5A, uncleaved) that upon cleavage, was reduced to a $27.4 \mathrm{kDa}$ protein composed of GFP and a few amino acids from the cleavage sequence (Fig. 5A, cleaved). Protease cleavage was observed at 14, 18 and $22 \mathrm{hrs,} \mathrm{with} \mathrm{optimum}$ observed after $18 \mathrm{hrs}$ of incubation.

Cleavage of mono-color type II MBR was also tested by Western blot analysis. With GAPDH as a masking protein and GFP fused to the C-terminus (see Fig. 1), this construct generated a $67.0 \mathrm{kDa}$ protein in the cell (Fig. 5B, uncleaved). After cleavage of the whole construct by HCV NS3 protease, the GAPDH masking protein is removed and GFP with the mitochondrial translocation signal at the $\mathrm{N}$-terminus is generated. The $\mathrm{N}$-terminal extension was lost upon transport into the mitochondria and GFP was detected in this organelle (Fig. 5B, cleaved). Once again, protease cleavage was at the experimental time points, 14, 18 and $22 \mathrm{hrs}$ with effective cleavage observed at all time points.

\section{Protease Inhibition Analysis with Known Inhibitors}

Our in-cell protease assay system was validated with a known HCV inhibitory compound. A peptide derivative, Ac-Asp-D-GlaLeu-Ile- $\beta$-cyclohexyl-Ala-Cyc-OH, which was reported as an HCV inhibitor [20] was used. The inhibitor was added to the cell culture medium during transfection and incubated with the cells as indicated. The number of cells with uncleaved and cleaved type I MBRs was counted by monitoring fluorescence localization changes inside living cells to obtain the MBR cleavage ratio at specific concentration of the protease inhibitor. As shown in Fig. 6A, a sigmoid curve was obtained. The half-maximal inhibitory concentration $\left(\mathrm{IC}_{50}\right)$ value was estimated to be approximately $28 \pm 7 \mathrm{nM}$. A similar graph was obtained with type II MBRs (Fig. 6B). In this case, the number of cells with dispersed fluorescence in the cytosol and with speckle-like fluorescence in mitochondria was counted to generate dose-response curve. The $\mathrm{IC}_{50}$ value was approximately $12 \pm 6 \mathrm{nM}$. The values obtained with these two different MBRs overlap each other within a 2 sigma error. The reported in vitro $\mathrm{IC}_{50}$ value of the inhibitor used was $40 \mathrm{nM}$ in $150 \mathrm{mM}$ of $\mathrm{NaCl}$ [20]. The $\mathrm{IC}_{50}$ values achieved in this study were lower than the reported in vitro value. The $\mathrm{NaCl}$ concentration used in this study was approximately $100 \mathrm{mM}$.

Inhibition of HCV NS3 protease was also examined with the incell-assay using a known RNA aptamer inhibitor [15]. Cells were co-transfected with vectors encoding type I MBR and HCV NS3 protease. Control cells were transfected with the MBR vector alone. The inhibitory RNA aptamer was added to the cell culture media during transfection at a concentration of approximately $7.5 \mu \mathrm{g} / \mathrm{mL}$. Cleavage of the expressed type I MBR was detected by measuring the intracellular fluorescence distribution after a 14hr incubation. As shown in Fig. 7, in the presence of inhibitory RNA aptamer, the inhibition rate was increased to approximately $35.8 \%$. The inhibition rate was lower than the reported in vitro efficiency of $90 \%$. However, considering drug delivery into the cell its intracellular stability, our results have significant meaning. We also found that the protease inhibiting activity was reduced over time (data not shown). These data imply that the RNA aptamer is degraded in the cell rapidly while the HCV protease was expressed continuously as the incubation time increases.

\section{Discussion}

There is no vaccine against HCV available yet and the major standard treatment for $\mathrm{HCV}$ is combination treatment of pegylated interferon alpha with ribavirin [21]. A few HCV protease inhibitors are undergoing clinical trials [22-24]. However, resistance to the current HCV protease inhibitors cures has been reported [25-26]. These circumstances put more weight on the importance of developing HCV anti-viral drug candidates and a rapid, sensitive assay for the enzyme. 


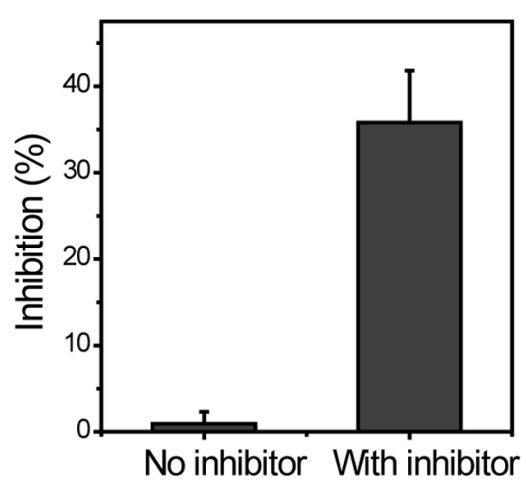

Figure 7. Inhibition of HCV NS3 protease by an RNA aptamer. An HCV NS3 protease inhibitory RNA aptamer was prepared and tested. Cells co-transfected with vectors encoding type I MBR and HCV NS3 protease were and treated with the RNA aptamer. The inhibition ratio was determined from the number of cells exhibiting a dispersed pattern relative to the total number of cells examined. doi:10.1371/journal.pone.0059710.g007

We developed an in-cell protease assay with HCV NS3 protease as a model system. We designed fluorescent molecular beacontype reporters that allow the translocation of reporter proteins to be monitored from one cellular compartment to another in living cells by simply observing the re-distribution of fluorescence. The in-cell assay system developed in this study is capable of measuring protease activity in a single cell in an on/off mode and can be used for quantitative analysis of protease inhibitors. In addition, since this new assay is performed with living cells, cell integrity, drug delivery into the cell, possible toxicity of candidate compounds and other relevant information can be monitored, which is very advantageous. Therefore, the assay developed here can be applied to high-content screening assays.

To acquire more statistically accurate data from the in-cell assay, it is important to secure an appropriate number of cells that show a clear image of fluorescent re-distribution before and after cleavage. Factors, including transfection efficiency, expression of

\section{References}

1. Ghosh AK, Brindisi M, Tang J (2012) Developing $\beta$-secretase inhibitors for treatment of Alzheimer's disease. J Neurochem 120: 71-83.

2. Adamson CS (2012) Protease-Mediated Maturation of HIV: Inhibitors of Protease and the Maturation Process. Mol Biol Int 2012: 604261.

3. Hook G, Hook V, Kindy M (2011) The cysteine protease inhibitor, E64d, reduces brain amyloid- $\beta$ and improves memory deficits in Alzheimer's disease animal models by inhibiting cathepsin B, but not BACE1, $\beta$-secretase activity. J Alzheimers Dis 26: 387-408.

4. Nimmanapalli R, Bhalla K (2003) Targets in apoptosis signaling: promise of selective anticancer therapy. Methods Mol Biol 223: 465-483.

5. Watson P (2009) Watson Live cell imaging for target and drug discovery. Drug News Perspect 22: 69-79.

6. Korn K, Krausz E (2007) Cell-based high-content screening of small-molecule libraries. Curr Opin Chem Biol 11: 503-510.

7. Fang Y, Frutos AG, Verklereen R (2008) Label-free cell-based assays for GPCR screening. Comb Chem High Throughput Screen 11: 357-369.

8. Smotrov N, Mathur A, Kariv I, Moxham CM, Bays N (2009) Development of a cell-based assay for measurement of c-Met phosphorylation using AlphaScreen technology and high-content imaging analysis. J Biomol Screen 14: 404-411.

9. Emery A, Sorrell DA, Lawrence S, Easthope E, Stockdale M, et al (2011) A novel cell-based, high-content assay for phosphorylation of Lats2 by Aurora A. J Biomol Screen 16: 925-931.

10. Yan YX, Boldt-Houle DM, Tillotson BP, Gee MA, D'Eon BJ, et al (2002) Cellbased high-throughput screening assay system for monitoring $\mathrm{G}$ protein-coupled receptor activation using beta-galactosidase enzyme complementation technology. J Biomol Screen 7: 451-459.

11. Choo QL, Kuo G, Weiner AJ, Overby LR, Bradley DW, et al (1989) Isolation of cDNA clone derived from a blood-borne non-A, non-B viral hepatitis genome. Science 244: 359-362. the MBRs, and incubation time before analysis of protease activity, are important and need to be considered [27-29]. We tried different transfection reagents, cell lines, translocation signals, expression vectors, and incubation times. To obtain statistically significant data, we counted more than 500 cells in each experiment and at least three independent experiments were performed for each assay. The data reported in this study was obtained using experimentally derived optimal conditions and results.

Another advantage of this assay is that various MBRs can be designed for assays to screen for inhibitors to a variety of proteases including other viral proteases, physiologically important proteases involved in other diseases, and signaling pathways. When applying the in-cell protease assay to other proteases, the minimum C.S length recognized by the target protease can be identified and inserted into MBRs. We tested different CS lengths for HCV in this study and found that a C.S with 10 amino acids, which is slightly longer than the reported consensus CS of 7 amino acids, yielded good cleavage [13]. It seems that space for effective protease binding is needed and must be considered when constructing MBRs for other proteases. Relatively longer CSs can be easily accommodated for in MBRs by a simple cloning process. Our new assay can also be applied to an HTS system. For additional applications, stable cell lines expressing an MBR and/ or protease can be generated.

Along with its broad application possibilities, we demonstrated that this assay is easy and simple to perform and score the results. The cleavage efficiency of the MBRs developed in this study are highly sensitive, reaching approximately $90 \%$ cleavage. Therefore, this assay is very sensitive and can be applied to inhibitor screening without the need for complex statistical tools.

\section{Author Contributions}

Conceived and designed the experiments: JHK IH HJH. Performed the experiments: JHK MJL HJH. Analyzed the data: JHK MJL HJH. Contributed reagents/materials/analysis tools: JHK MJL HJH. Wrote the paper: JHK.
12. Choo QL, Richman KH, Han JH, Berger K, Lee C, et al (1991) Genetic organization and diversity of the hepatitis C virus. Proc Nat Acad Sci 88: 24512455.

13. Tan SL (2006) Hepatitis C Viruses: Genomes and Molecular Biology. Horizon Bioscience.

14. Piao W, Yoo J, Lee DK, Hwang HJ, Kim JH (2001) Induction of G(2)/M phase arrest and apoptosis by a new synthetic anti-cancer agent, DW2282, in promyelocytic leukemia (HL-60) cells. Biochem Pharmacol 62: 1439-1447.

15. Fukuda K, Vishnuvardhan D, Sekiya S, Hwang JS, Kakiuchi N, et al (2000) Isolation and characterization of RNA aptamers specific for the hepatitis $\mathrm{C}$ virus nonstructural protein 3 protease. Eur J Biochem 267: 3685-3694.

16. Harlan JE, Hajduk PJ, Yoon HS, Fesik SW (1994) Pleckstrin homology domains bind to phosphatidylinositol-4,5-bisphosphate. Nature 371: 168-170.

17. Holz RW, Hlubek MD, Sorensen SD, Fisher SK, Balla T, et al (2000) A pleckstrin homology domain specific for phosphatidylinositol 4, 5-bisphosphate (PtdIns-4,5-P2) and fused to green fluorescent protein identifies plasma membrane PtdIns-4,5-P2 as being important in exocytosis. J Biol Chem 275: $17878-17885$.

18. Kuschel L, Hansel A, Schonherr R, Weissbach H, Brot N, et al (1999) Molecular cloning and functional expression of a human peptide methionine sulfoxide reductase (hMsrA). FEBS 456: 17-21.

19. Hansel A, Kuschel L, Hehl S, Lemke C, Agricola HJ, et al (2002) Mitochondrial targeting of the human peptide methionine sulfoxide reductase (MSRA), an enzyme involved in the repair of oxidized proteins. FASEB 16: 911-913.

20. Ingallinella P, Altamura S, Bianchi E, Taliani M, Ingenito R, et al (1998) Potent peptide inhibitors of human hepatitis $\mathrm{C}$ virus NS3 protease are obtained by optimizing the cleavage products. Biochem 37: 8906-8914.

21. Shepherd J, Brodin H, Cave C, Waugh N, Price A, et al (2004) Pegylated interferon alpha- $2 \mathrm{a}$ and $-2 \mathrm{~b}$ in combination with ribavirin in the treatment of 
chronic hepatitis C: a systematic review and economic evaluation. Health Technol Assess. 8: 1-125.

22. Poordad F, McCone J Jr, Bacon BR, Bruno S, Manns MP, et al (2011) Boceprevir for untreated chronic HCV genotype 1 infection. N Engl J Med 364: 1195-1206.

23. Bacon BR, Gordon SC, Lawitz E, Marcellin P, Vierling JM, et al (2011) Boceprevir for previously treated chronic HCV genotype 1 infection. N Engl J Med 364: 1207-1217.

24. Forestier N, Zeuzem S (2012) Telaprevir for the treatment of hepatitis C. Expert Opin Pharmacother. 13: 593-606.

25. Halfon P, Locarnini S (2011) Hepatitis G virus resistance to protease inhibitors. J Hepatol 55: 192-206.
26. Welsch C, Shimakami T, Hartmann C, Yang Y, Domingues FS, et al (2012) Peptidomimetic escape mechanisms arise via genetic diversity in the ligandbinding site of the hepatitis C virus NS3/4A serine protease. Gastroenterology. 142: 654-663.

27. Paecharoenchai O, Niyomtham N, Ngawhirunpat T, et al (2012) Cationic niosomes composed of spermine-based cationic lipids mediate high gene transfection efficiency. J Drug Target 29: 783-792.

28. Hasemi A, Roohvand F, Ghahremani MH, et al (2012) Optimization of transfection methods for Huh-7 and vero celss: a comparative study. Tsitol Genet 46: 19-27.

29. Guan L, Sun Y, Xi Q et al (2013) b-Glucanase specific expression in the parotid gland of transgenic mice. Transgenic Res DOI 10.1007/s11248-012-9682-3. 